\title{
Impacts of albumin synergized with hydroxyethyl starch on early microvascular albumin leakage after major abdominal surgery in rabbits ${ }^{1}$
}

\begin{abstract}
Weiming Li', Pengyuan Xu", Yunyun Cen"', Dali Sunv, Ting Yang ${ }^{\vee}$, Qingwen Xu , Shumin Li, Yijun Li, Bo Ding ${ }^{\circ}$

'PhD, General Surgeon, Department of Gastrointestinal Surgery, the Second Affiliated Hospital, Kunming Medical University, China. Conception and design of the study, technical procedures, acquisition and analysis of data, manuscript writing. "Professor, General Surgeon, Department of Gastrointestinal Surgery, the Second Affiliated Hospital, Kunming Medical University, China. Conception, design, intellectual and scientific content of the study; critical revision; final approval the manuscript.

I'Professor, General Surgeon, Department of Gastrointestinal Surgery, the Second Affiliated Hospital, Kunming Medical University, China. Analysis and interpretation of data.

IVMD, General Surgeon, Department of Gastrointestinal Surgery, the Second Affiliated Hospital, Kunming Medical University, China. Acquisition of data.

${ }^{\vee} \mathrm{MD}$, General Surgeon, Department of Gastrointestinal Surgery, the Second Affiliated Hospital, Kunming Medical University, China. Design of the study, acquisition and interpretation of data, technical procedures.
\end{abstract}

\section{Abstract}

Purpose: To investigate the impacts of albumin synergized with hydroxyethyl starch (HES) on early microvascular albumin leakage after major abdominal surgery in rabbits.

Methods: Forty male Japanese rabbits were randomly divided into four groups: the control group, the saline group, the albumin group, and the Syn group (hydroxyethyl starch+albumin). The latter three groups were performed gastrectomy plus resection of pancreatic body and tail and splenectomy. The serum albumin concentration was detected before and $48 \mathrm{~h}$ after surgery, and the conditions of mesenteric microvascular leakage in these 4 groups were observed under microscope $48 \mathrm{~h}$ after surgery to calculate the leakage rate.

Results: Compared with the saline group, the albumin group and the Syn group exhibited significantly increased serum albumin concentrations $48 \mathrm{~h}$ after surgery $(P<0.05)$. The albumin leakage rate was the most obvious in the albumin group, followed by the saline group, while that in the Syn group was the minimal, and there existed significant differences among these groups $(\mathrm{P}<0.05)$.

Conclusion: Simple administration of albumin in the early stage after major abdominal surgery could increase the albumin leakage, while the synergization of albumin and hydroxyethyl starch could reduce the albumin leakage.

Key words: Hydroxyethyl Starch Derivates. Albumins. Rabbits. 


\section{- Introduction}

It was found in clinical studies that: there commonly existed the phenomenon of serum albumin decreasing after severe trauma, infection and major abdominal surgery $^{1-3}$. Hypoproteinemia could decrease intravascular colloid osmotic pressure, so that the wound and other organs/tissues would exhibit edema, which would not only affect body's wound healing and recovery, but also increase postoperative pulmonary edema, anastomotic stoma fistula, wound disunion, infections and other complications, and the mortality rate might be increased ${ }^{4-6}$. Studies had found that the decreasing of serum albumin levels often indicated poor prognosis towards critically ill patients. ${ }^{7-9}$ So, how to prevent and treat hypoalbuminemia had been a focus of the study, the administration of exogenous albumin seemed very reasonable to correct hypoalbuminemia, but there existed a lot of controversy in clinical practices that whether the infusion of exogenous albumin towards patients with hypoalbuminemia could improve their prognosis. Hydroxyethyl starch 130/0.4 (HES) was the thirdgeneration artificial colloid applied clinically, and its roles in preventing microvascular albumin leakage had obtained more and more attentions from researchers. Due to the limitations of experimental techniques and ethics, it was difficult to carry out the experiments to directly observe the impacts of HES on microvascular albumin leakage in human, our experiments used rabbits as the subjects, used fluorescent protein tracer technique, as well as confocal laser scanning microscopy (CLSM) which could perform accurate quantitative analysis ${ }^{10}$, to directly detect the tracer albumin leakage, therefore deducing the synergic impacts of albumin and HES on early microvascular albumin leakage after major abdominal surgery in rabbits.

\section{- Methods}

This study was carried out in strict accordance with the recommendations in the Guide for the Care and Use of Laboratory Animals of the National Institutes of Health. The animal use protocol has been reviewed and approved by the Institutional Animal Care and Use Committee (IACUC) of Kunming Medical University.

Forty healthy Japanese rabbits, weighed $2.5 \pm 0.5 \mathrm{~kg}$, male, were provided by the Experimental Animal Center of Kunming Medical University (License No. SYXK (Yunnan) 2011-0004).

\section{Experimental methods}

These forty healthy male Japanese rabbits were randomly divided into the control group, the saline group, the albumin group, the Syn (HES+albumin) group, with 10 rabbits in each group. The control group was not performed surgery, while the latter three groups were performed gastrectomy, together with resection of pancreatic body and tail and splenectomy, the surgical procedures were performed by the same staff, and the operation time was strictly controlled within 1.5 h. Each rabbit was adopted isocaloric, isonitric nutritional support postoperative after operation. Total calories of each rabbit was 150 kcal daily. Glucose and fat offered $70 \%$ and $30 \%$ of the calories respectively. The ratio of nitrogen and calorie was 1:130. After surgery, the saline group was given 25 $\mathrm{ml} / \mathrm{kg}$ physiological saline; the albumin group was given $5 \%$ rabbit serum albumin $25 \mathrm{ml} / \mathrm{kg}$; the Syn group was given $25 \mathrm{ml} / \mathrm{kg}$ liquid that included 6\% HES $12.5 \mathrm{ml} / \mathrm{kg}$ and 5\% rabbit serum albumin $12.5 \mathrm{ml} / \mathrm{kg}$. The control group was considered as preoperative indicators. 


\section{Anaesthesia, gastrectomy, and postoperative care}

The gastrectomy was performed. In brief, after a $24 \mathrm{~h}$ fast, all the rabbits were subjected to laparotomy under pentobarbital anesthesia. The greater omentum was resected around the pyloric region, and the pancreatic head and body were exposed. The pancreas was freed at the junction of the head and body, while avoiding damage to the bile duct, portal vein and duodenal vessels, and the organ was ligated and cut at this site. Pancreaticogastrostomy was performed in the three groups. All rabbits were received about $250 \mathrm{ml}$ of lactated Ringer's solution intravenously during and just after surgery. Postoperatively, all rabbits were fasted and given saline ( $60 \mathrm{ml} /$ day) and $10 \%$ glucose (60 $\mathrm{ml} /$ day) by subcutaneous injection.

\section{Detection indictors}

The saline group, the albumin group and the Syn group: Preoperative fasting blood for $24 \mathrm{~h}$ postoperatively on an empty stomach were extracted, and the serum albumin concentration was detected using AU5841 biochemical analyzer (Beckman Coulter, Indianapolis, IN, USA). The control group, the saline group, the albumin group and the Syn group $48 \mathrm{~h}$ after surgery: Intravenously injected rhodamine isothiocyanate-labeled bovine serum albumin (5 $\mu \mathrm{mol} / \mathrm{L}$, SigmaAldrich, USA), then dynamically observed the mesenteric microvascular albumin leakage under CLSM (Olympus, Japan), and calculated the vascular permeability rate.

\section{Observation of microvascular albumin leakage under CLSM}

(1) Principle of laser confocal microscopy

Traditional optical microscope used light source, and image at each point was disturbed by diffraction or scattered light in proximal point. However, confocal laser scanning microscope could scan every point by formed point light source via laser beam, exposure point was imaged in detection pinhole and was received point-by-point or line-by-line by infrared photo multiplier or cold electrical coupling device. Fluorescence image was rapidly formed in computer. Lighting pinhole and detection pinhole were conjugated compared with the focal plane of the objective, and points in focal plane were simultaneously focused on lighting pinhole and launch pinhole. Point outside of focal plane was not imaged in detection pinhole, thus, obtained confocal images were optical cross section and avoided disadvantages of general microscope.

(2) Detailed methods

The rabbits of each group were fixed into the surgical box at each postoperative time point, then injected $3 \%$ pentobarbital sodium (30 mg/kg) through ear vein for anesthesia; when totally anesthetized, removed the rabbit from the surgical box, fixed on the operating table in the supine position, disinfected, paved sterile towels, and re-incised along the original incision. Fixed the rabbit under CLSM, lifted $15 \mathrm{~cm}$ of small intestine and intestinal loop for the naked-eye observation about the mesenteric vascular distributions, chose the part with small blood vessels distributed while less fat for the observation, stretched the pulled-out mesentery on the transparent plexiglass-made dish; chose excitation wavelength as $543 \mathrm{~nm}$, and the objective lens as 20x, injected rhodamine isothiocyanatelabeled bovine serum albumin solution $1 \mathrm{~mL} /$ $\mathrm{kg}$ into ear vein. Selected micro vessel with diameter as 20-35 $\mu \mathrm{m}$ to dynamically record mesenteric microvascular albumin leakage, and recorded the digitized images. Used FV10-ASW 2.1 Viewer software to measure 
and calculate the digital images obtained; selected 2 regions inside and outside the vessels to measure and calculate the average fluorescence intensity before and 2 min after the injection of rhodamine isothiocyanatelabeled bovine serum albumin, the actual fluorescence intensity = fluorescence intensity after injection-fluorescence intensity before injection. Calculated the relative change of mesenteric inner and extra-microvascular fluorescence intensity, which was used to represent the mesenteric microvascular albumin leakage ${ }^{11}: \Delta \mathrm{I}=1-(\mathrm{I}-\mathrm{IO}) / \mathrm{li}(\Delta \mathrm{I}$ : relative change of fluorescence intensity, Ii: intravascular fluorescence intensity, 10: extravascular fluorescence intensity).

\section{Statistical processing}

All data were performed analysis of variance and multiple comparison (LSD) with statistical software SPSS17.0 (SPSS Inc., Chicago, IL, USA), the data were expressed with mean \pm standard deviation $(\bar{X} \pm s)$.

\section{- Results}

\section{Comparison of serum albumin concentration}

There was no statistically significant difference in serum albumin concentration among the saline group, the albumin group and the Syn group before surgery $(P>0.05)$, while the concentrations of these 3 groups were decreased after surgery, and the differences were significant than those before surgery $(P<0.05)$. The comparison of serum albumin concentration $48 \mathrm{~h}$ after surgery showed that, the albumin group and the Syn group were significantly increased than the saline group $(P<0.05)$, while there was no significant difference between the albumin group and the Syn group (Table 1).

Table 1 - Comparison of serum albumin in three groups (g/L) $(n=10, \bar{X} \pm s)$.

\begin{tabular}{lcc}
\hline Group & Before surgery & After surgery \\
Saline group & $41.21 \pm 4.83$ & $30.47 \pm 4.44 \#$ \\
Albumin group & $41.53 \pm 3.32$ & $36.31 \pm 4.36^{*} \#$ \\
\hline Syn group & $40.97 \pm 4.24$ & $36.57 \pm 4.01^{*} \#$ \\
\hline
\end{tabular}

\#Compared with that before surgery; *Compared with the saline group $(p<0.05)$.

Comparison of mesenteric microvascular albumin leakage $48 \mathrm{~h}$ after major abdominal surgery

Compared with the control group, the mesenteric microvessels of the saline group, the albumin group and the Syn group all exhibited mesenteric microvascular albumin leakage $48 \mathrm{~h}$ after major abdominal surgery.
While the comparison among the saline group, the albumin group and the Syn group showed that the Syn group had the minimal microvascular albumin leakage, while the albumin group had the maximal leakage, and the comparison among the 3 groups had significant difference $(\mathrm{P}<0.05)$ (Figures 1 and 2). 


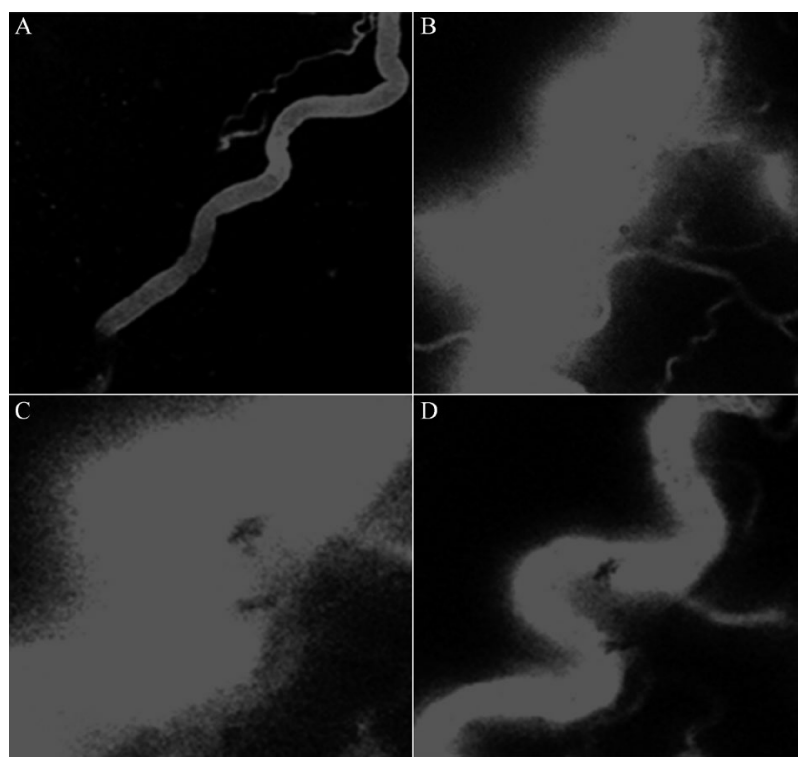

Figure 1 - Comparison of mesenteric microvascular albumin leakage (48h) after major abdominal surgery among the four groups. A: Control group; B: Saline group; C: Albumin group; D: Syn group.

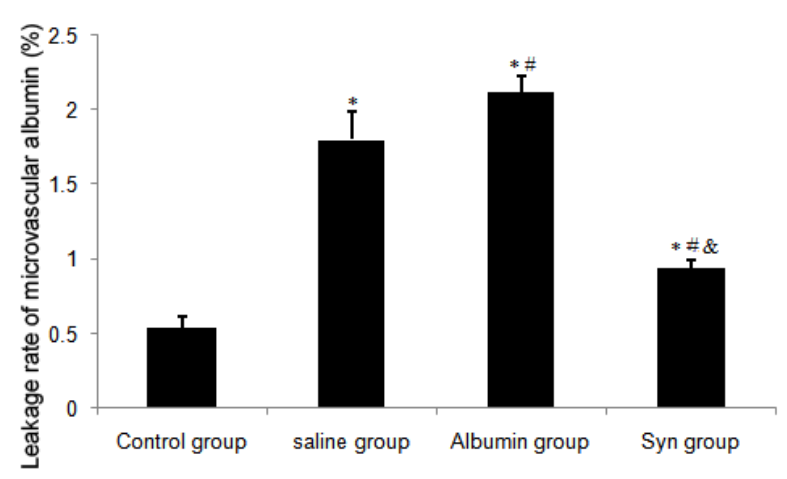

Figure 2 - Comparison of leakage rate of microvascular albumin postoperative $48 \mathrm{~h}(\mathrm{n}=10)$. *Means compared with the control group,\# saline group, and albumin group $(P<0.05)$.

\section{- Discussion}

There commonly existed the phenomenon that serum albumin concentration was acutely decreased after major surgery or severe trauma clinically ${ }^{1-3}$, as the most important ingredient to maintain plasma colloid osmotic pressure, albumin could not only regulate dynamic balance of water between tissues and vessels, but also had such important effects as antioxidation, transporting hemoglobin, hormones and fatty acids, etc, the degree of hypoproteinemia was positively correlated with the patients' complications and mortality ${ }^{12-14}$. How to correct hypoproteinemia? The traditional view believed that the supplement of exogenous albumin was the best way. A multicenter, random and double-blind study towards 6997 critically ill patients and patients after surgery showed that 15 : the $4 \%$ albumin group and the saline group no significant difference in intensive care unit (ICU) time, length of hospital stay, mechanical ventilation time, renal replacement therapy rate, and so on, and the research towards different subgroups showed that the mortality rate in the albumin group of trauma patients was slightly higher.

In recent years, some scholars had proposed that the major surgery or severe traumatic stress-caused tissue or systemic inflammatory response-led microvascular albumin leakage redistributed albumin inside and outside blood vessels, which was main reason towards the early declining of serum albumin concentration ${ }^{16,17}$. So it was worthy of studying the impacts of early albumin application on microvascular albumin leakage after major abdominal surgery. Currently, the main methods to reduce microvascular albumin leakage included: controlling infection, improving intravascular osmotic pressure through applying hypertonic saline or artificial colloid, applying inflammatory mediator antagonists, etc, among which HES 130/0.4 was one kind of novel HES with middle molecular weight, which could reduce vascular endothelial cell injuries and neutrophil-mediated tissue damages, increase intravascular osmotic pressure, reduce inflammatory responses, and maintain oxygenation of blood system, thus improving microcirculation and reducing microvascular leakage ${ }^{18}$, HES 130/0.4 also had certain "sealing" effects towards microvascular leakage ${ }^{19,20}$. Therefore, this study synergized 
albumin and HES to observe the impacts on mesenteric microvascular albumin leakage in the early stage after major abdominal surgery.

Detection of leakage rate of microvascular albumin is an important method to estimate microvascular permeability and leakage of albumin. There are two methods to detect leakage rate: 1) detection extracellular fluid after intravenous infusion of exogenous albumin: observe changes of colloid osmotic concentration using inulin distribution capacity and bio-electrical impedance ${ }^{21}$. This method is safety and noninvasive, but it is expensive and is not adapt to popularization and application in clinic and basic study. 2) Fluorescein labeled albumin: albumin is fluorescence labeled via intravenous fluorescent, and leakage rate is estimated using intensity changes of relative fluorescence of the blood vessels inside and outside using fluorescence microscope ${ }^{22,23}$. In this study, rhodamine isothiocyanate is used to label bovine serum albumin to form RBITC-BSA, which is used to estimate leakage rate via observing leakage phenomenon of microvascular albumin in postoperative rabbit abdominal mesenteric. Our study aims to discuss effect of major abdominal surgery on leakage of microvascular albumin in rabbit mesentery. Due to the lighting pinhole of laser confocal microscope and detecting pinhole are conjugated compared to lens focal plane, the points on the focal plane simultaneously focus on illumination pinhole and launch pinhole, and other points are not imaged in detection pinhole. Thus, obtained confocal images are optical cross section of samples, which avoids disadvantages of blurry images in general microscope and the images are clear, visual, easy to operate and safety.

VThe results of this experiment showed: the serum albumin levels of the 3 groups were significantly reduced after major surgery lower than those before surgery, while the serum albumin values of the albumin group and the Syn group $48 \mathrm{~h}$ after surgery were significantly increased than the saline group $(P<0.05)$.
Therefore, it could be inferred that there existed the declining of serum albumin in early stage after major abdominal surgery, and the synergic infusion of albumin and HES could improve serum albumin concentration.

Our experiments used fluorescencelabeling technology and CLSM to track and observe the tracing albumin, which could clearly, intuitively, and accurately observe the whole process that the tracing albumin escaped from the leaking sites among endothelial cell gaps to the outside tissues, and the image analysis system could performed precise quantitation to calculate the impacts of albumin, synergized with HES, and saline on early microvascular albumin leakage in rabbits after major abdominal surgery, respectively. The present results showed that $48 \mathrm{~h}$ after major abdominal surgery, there existed microvascular albumin leakage in the saline group, the albumin group and the Syn group, but the leakage of the albumin group was the most obvious, which might probably be the main disadvantage that affected the prognosis after major abdominal surgery. The Syn group exhibited the lightest microvascular albumin leakage; meanwhile, it also played certain "blocking" effects towards microvascular leakage, reduced microvascular albumin leakage, reduced the amount of albumin, thus improving human serum albumin concentration and the prognosis.

\section{- Conclusion}

The present study provided the theoretical basis for the proper use of albumin and early microvascular albumin leakage prevention after major abdominal surgery. The study could also prevent the abuse of albumin, save medical resources and improve patients' prognosis.

\section{- References}

1. Alberti LR, Petroianu A, Zac RI, Andrade $J C$ Jr. The effect of surgical procedures on 
serum albumin concentration. Chirurgia (Bucur). 2008 Jan-Feb;103(1):39-43. PMID: 18459495.

2. Kudsk KA, Tolley EA, DeWitt RC, Janu PG, Blackwell AP, Yeary S, King BK. Preoperative albumin and surgical site identify surgical risk for major postoperative complications. JPEN J Parenter Enteral Nutr. 2003 JanFeb;27(1):1-9. PMID: 12549591.

3. Koertzen M, Punjabi P, Lockwood G. Preoperative serum albumin concentration as a predictor of mortality and morbidity following cardiac surgery. Perfusion. 2013 Sep;28(5):390-4. doi: 10.1177/0267659113488990.

4. Nakamura K, Igarashi K, Ide K, Ohkawa R, Okubo S, Yokota H, Masuda A, Oshima N, Takeuchi T, Nangaku M, Okudaira S, Arai $\mathrm{H}$, Ikeda $\mathrm{H}$, Aoki J, Yatomi Y. Validation of an autotaxin enzyme immunoassay in human serum samples and its application to hypoalbuminemia differentiation. Clin Chim Acta. 2008 Feb;388(1-2):51-8. doi: 10.1016/j.cca.2007.10.005.

5. Lee JI, Kwon M, Roh JL, Choi JW, Choi SH, Nam SY, Kim SY. Postoperative hypoalbuminemia as a risk factor for surgical site infection after oral cancer surgery. Oral Dis. 2015 Mar;21(2):178-84. doi: 10.1111/odi.12232.

6. Adogwa O, Martin JR, Huang K, Verla T, Fatemi P, Thompson P, Cheng J, Kuchibhatla M, Lad SP, Bagley CA, Gottfried ON. Preoperative serum albumin level as a predictor of postoperative complication after spine fusion. Spine (Phila Pa 1976). 2014 Aug 15;39(18):1513-9. doi: 10.1097/ BRS.0000000000000450.

7. Leite HP, Fisberg M, de Carvalho WB, de Camargo Carvalho AC. Serum albumin and clinical outcome in pediatric cardiac surgery. Nutrition. 2005 May;21(5):553-8. doi: 10.1016/j.nut.2004.08.026.

8. Borda F, Borda A, Jiménez J, Zozaya JM, Prieto C, Gómez M, Urman J, Ibáñez B. Predictive value of pre-treatment hypoalbuminemia in prognosis of resected colorectal cancer. Gastroenterol Hepatol. 2014 May;37(5):28995. doi: 10.1016/j.gastrohep.2013.12.007.

9. González Infantino CA, González CD,
Sánchez R, Presner N. Hyperglycemia and hypoalbuminemia as prognostic mortality factors in patients with enteral feeding. Nutrition. 2013 Mar;29(3):497-501. doi: 10.1016/j.nut.2012.07.019.

10. Wang P, Li Y, Li J. Hydroxyethyl starch $130 / 0.4$ prevents the early pulmonary inflammatory response and oxidative stress after hemorrhagic shock and resuscitation in rats. Int Immunopharmacol. 2009 Mar;9(3):34753. doi: 10.1016/j.intimp.2008.12.014.

11. Childs EW, Udobi KF, Hunter FA, Dhevan V. Evidence of transcellular albumin transport after hemorrhagic shock. Shock. 2005 Jun;23(6):565-70. PMID: 15897811.

12. Famakin B, Weiss $P$, Hertzberg V, McClellan W, Presley R, Krompf K, Karp H, Frankel MR. Hypoalbuminemia predicts acute stroke mortality: Paul Coverdell Georgia Stroke Registry. J Stroke Cerebrovasc Dis. 2010 Jan;19(1):17-22. doi: 10.1016/j. jstrokecerebrovasdis.2009.01.015.

13. Baldwin MR, Arcasoy SM, Shah A, Schulze PC, Sze J, Sonett JR, Lederer DJ. Hypoalbuminemia and early mortality after lung transplantation: a cohort study. Am J Transplant. 2012 May;12(5):1256-67. doi: 10.1111/j.1600-6143.2011.03965.x.

14. Foley RN, Parfrey PS, Harnett JD, Kent GM, Murray DC, Barre PE. Hypoalbuminemia, cardiac morbidity, and mortality in endstage renal disease. J Am Soc Nephrol. 1996 May;7(5):728-36. PMID: 8738808.

15. Finfer $\mathrm{S}$, Bellomo $\mathrm{R}$, Boyce $\mathrm{N}$, French J, Myburgh J, Norton R; SAFE Study Investigators. A comparison of albumin and saline for fluid resuscitation in the intensive care unit. N Engl J Med. 2004 May 27;350(22):2247-56. doi: 10.1056/ NEJMoa040232.

16. Uhing MR. The albumin controversy. Clin Perinatol. 2004 Sep;31(3):475-88. doi: 10.1016/j.clp.2004.03.018.

17. Gill SE, Taneja R, Rohan M, Wang L, Mehta S. Pulmonary microvascular albumin leak is associated with endothelial cell death in murine sepsis-induced lung injury in vivo. PLoS One. 2014 Feb 7;9(2):e88501. doi: 10.1371/journal.pone.0088501. 
18. Hiltebrand LB1, Kimberger O, Arnberger M, Brandt S, Kurz A, Sigurdsson GH. Crystalloids versus colloids for goal-directed fluid therapy in major surgery. Crit Care. 2009;13(2):R40. doi: $10.1186 / c c 7761$.

19. Marx G, Cobas Meyer M, Schuerholz T, Vangerow B, Gratz KF, Hecker H, Sümpelmann $\mathrm{R}$, Rueckoldt $\mathrm{H}$, Leuwer M. Hydroxyethy starch and modified fluid gelatin maintain plasma volume in a porcine model of septic shock with capillary leakage. Intensive Care Med. 2002 May;28(5):629-35. doi: 10.1007/ s00134-002-1260-3.

20. Lv R, Zhou W, Chu C, Xu J. Mechanism of the effect of hydroxyethyl starch on reducing pulmonary capillary permeability in a rat model of sepsis. Ann Clin Lab Sci. 2005 Spring;35(2):174-83. PMID: 15943182.
21. Marx G, Vangerow B, Burczyk C, Gratz $\mathrm{KF}$, Maassen N, Meyer MC. Evaluation of noninvasive determinants for capillary leakage syndrome in septic shock patients. Intensive Care Med. 2000 Sep;26(9):1252-8. doi: $10.1007 / \mathrm{s} 001340000601$.

22. Chen HM, Sunamura $M$, Shibuya $K$, Yamauchi JI, Sakai Y, Fukuyama S. Early microcirculatory derangement in mild and severe pancreatitis models in mice. Surgery Today. 2001;31(7):634-42. doi: 10.1007/ s005950170098.

23. Eibl G, Hotz HG, Faulhaber J, Kirchengast M, Buhr HJ, Foitzik T. Effect of endothelin and endothelin receptor blockade on capillary permeability in experimental pancreatitis. Gut. 2000; 46(3): 390-4. doi: 10.1136/ gut.46.3.390.

\section{Correspondence:}

Pengyuan $\mathrm{Xu}$,

Department of Gastrointestinal Surgery

The Second Affiliated Hospital of Kunming Medical University

Kunming 650101, China

Phone/Fax: +8687163402278

pengyuanxudoc@163.com

Conflict of interest: none

Financial sources: National Natural Science Foundation of China (project number: 81160114), Joint Special Projects of Yunnan Provincial Science and Technology Department (2011FB196)
Received: Oct 04, 2016

Review: Dec 07, 2016

Accepted: Jan 09, 2017

${ }^{1}$ Research performed at Department of Gastrointestinal Surgery, The Second Affiliated Hospital, Kunming Medical University, China. 